\title{
SIMULATION STUDY OF HIGHER-ORDER-MODE DAMPING OF STORAGE RING CAVITIES
}

\author{
C. Sung, K.R. Chu, Department of Physics, National Tsing Hua University \\ Y.C. Tsai, Electronics Research and Service Organization, Industrial Technology Research Institute \\ Ch. Wang, Synchrotron Radiation Research Center, Hsinchu, Taiwan \\ E. Weihreter, BESSY, Berlin, Germany
}

\begin{abstract}
We present studies of a higher-order-mode damping device, which employs a circular waveguide to coaxial transition (CWCT). The waveguide is double-ridged. A properly shaped back cavity at the far end allows broadband coupling to a coaxial window. Configurations of CWCTs with either symmetric or asymmetric ridge depth are both optimized with the HFSS code. HFSS calculations have also been performed to examine CWCT damping effects on a pillbox cavity. The lowest order anti-symmetric modes are shown to be much more effectively damped by the asymmetric CWCT. However, relative merits of the symmetric and asymmetric CWCTs depend on other factors such as the cavity configuration.
\end{abstract}

\section{INTRODUCTION}

The concept of the waveguide filter [1] has been extensively exploited for the damping of HOMs of the storage ring cavities. A circular cross-section version of the damping device (CWCT) was proposed in [2] and studied in [2-5]. In its original configuration, the doubleridged waveguide of the CWCT was symmetric in ridge width and depth in the cross-sectional plane (Fig. 2a). When located in the middle section of the sidewall of the cavity, it is effective in damping the modes with even axial mode indexes $(l)$ but produces very little damping effects on modes with odd mode indexes ([4] and Fig. 1). In [6], it was shown that the $\mathrm{TM}_{011}$ mode could be damped to $\mathrm{Q} \approx 1000$ by a $30 \mathrm{~mm}$ axial shift of the symmetric CWCT away from the middle plane of the cavity, which suggests that the odd- $l$ modes could also be effectively damped by a CWCT with asymmetric ridges (Fig. 2b). Furthermore, as shown in [6], the radius of an asymmetric CWCT is smaller than that of the symmetric CWCT with the same cutoff frequency (see Fig. 2).

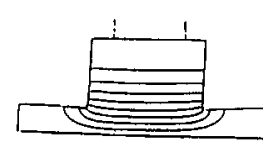

(a)

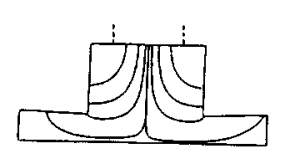

(b)

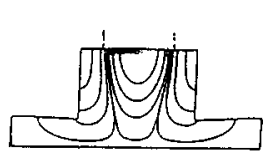

(c)
Fig. 1. Field patterns of modes with different axial mode indexes: (a) $l=0$, (b) $l=1$, and (c) $l=2$.
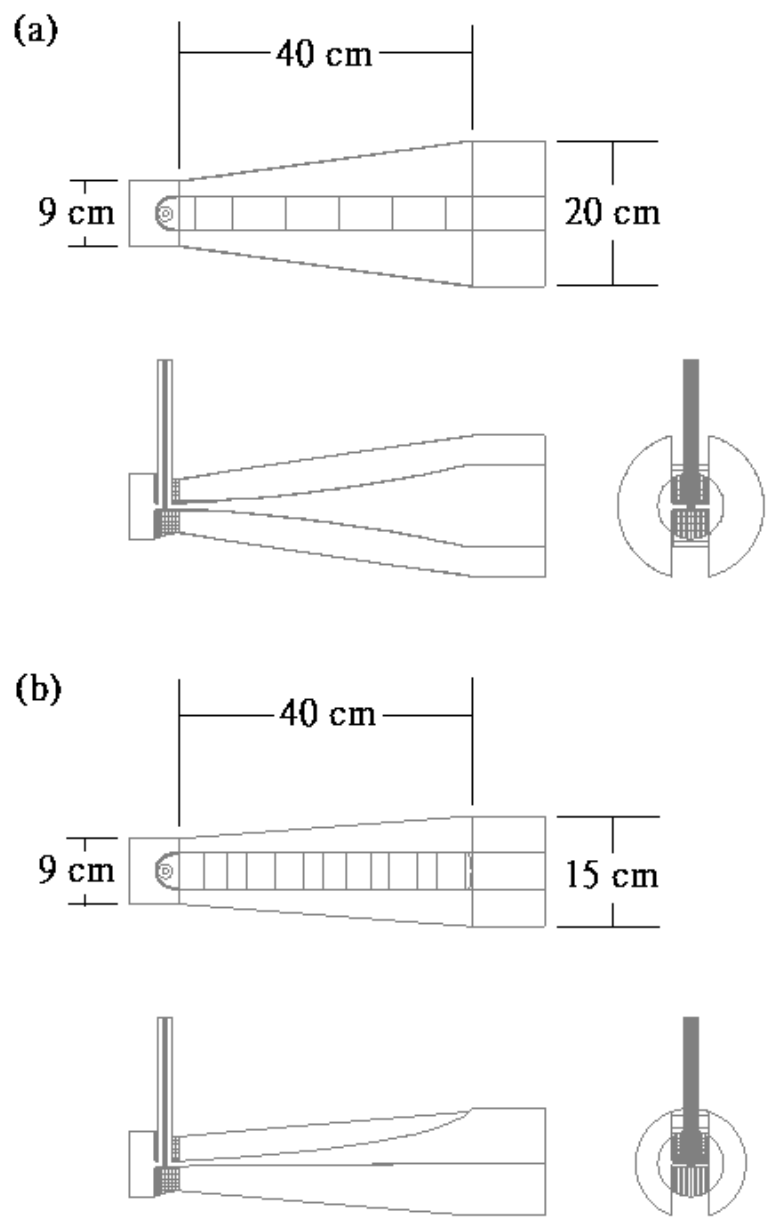

Fig. 2. Configuration of the (a) symmetric and (b) asymmetric CWCT.

In this report, we employ the HFSS code to examine the damping of HOMs of the DORIS cavity by a recently designed asymmetric CWCT [6] shown in Fig. 2b. Outgoing-wave boundary condition is imposed at the tip of the coaxial section. In order to search for the high Q modes in the frequency domain, we have employed a three-point extrapolation method [7], which allows us to find the resonant frequency and Q value of a high Q mode with only a few iterations. For the purpose of comparison, damping effects of a symmetric CWCT [4] are also examined. The $S_{11}$ parameters of the symmetric and asymmetric CWCTs are shown in Fig. 3. 


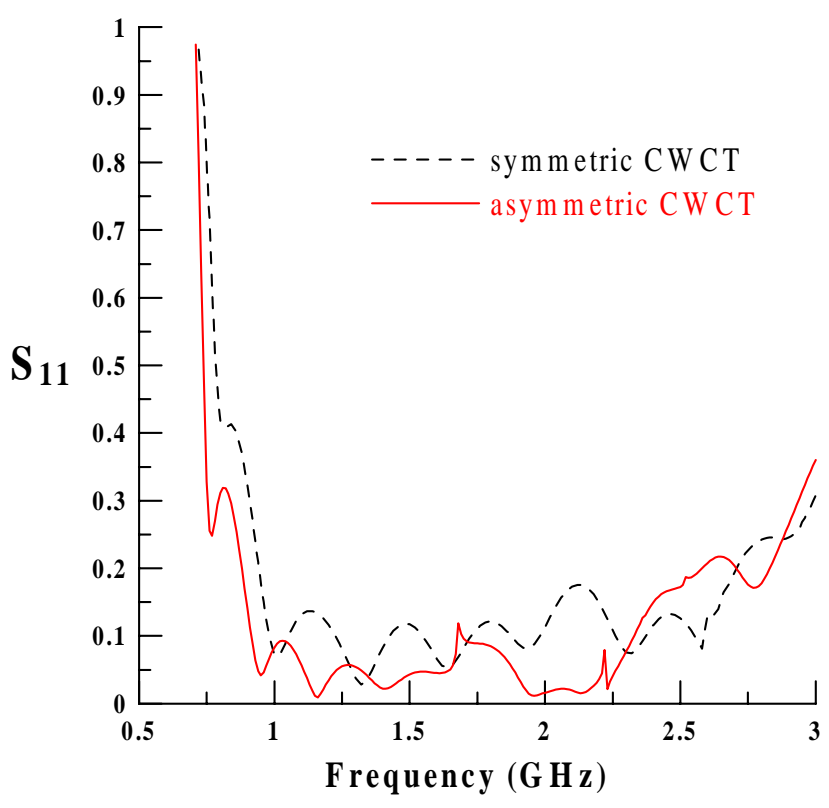

Fig. 3. HFSS simulation of the $S_{11}$ parameters of the (a) symmetric and (b) asymmetric CWCTs.

\section{RESULTS}

The calculated damping effects of a single asymmetric CWCT on the first few modes of the DORIS cavity is shown in Fig. 4 and compared with those of a symmetric damper. Main observations are summarized below:

1. The operating $\left(\mathrm{TM}_{010}\right)$ mode is almost unaffected by either the symmetric or asymmetric CWCT because the operating mode frequency $(500 \mathrm{MHz})$ is below the cutoff frequency $(710 \mathrm{MHz})$ of the CWCTs.

2. Damping effects on the other $l=0$ modes $\left(\mathrm{TM}_{110}\right.$, $\mathrm{TM}_{210}$, and $\left.\mathrm{TM}_{020}\right)$ are significant with either CWCT. The symmetric CWCT is more effective in damping the $\mathrm{TM}_{210}$ and $\mathrm{TM}_{020}$ modes because the field patterns of these modes near the aperture is more compatible to the fundamental propagating mode of the symmetric CWCT than those of the asymmetric CWCT.

3. The $l=1$ modes $\left(\mathrm{TM}_{011}, \mathrm{TM}_{111}\right.$, and $\left.\mathrm{TM}_{211}\right)$, which can't be damped by the symmetric CWCT, are effectively damped by the asymmetric CWCT to $\mathrm{Q} \approx 500$ for the $\mathrm{TM}_{011}$ and $\mathrm{TM}_{111}$ modes and $\mathrm{Q} \approx 1500$ for the $\mathrm{TM}_{211}$ mode.

4. Damping effects on the $l=2$ modes $\left(\mathrm{TM}_{112}\right.$ and $\mathrm{TM}_{012}$ ) are significant with either damper. The symmetric CWCT are more effective on the $\mathrm{TM}_{012}$ and $\mathrm{TM}_{112}$ modes than the asymmetric one, because the aperture of the asymmetric CWCT falls in a field pattern less favorable for coupling.

Fig. 4 was obtained with the asymmetric CWCT placed in the middle of the sidewall. When the asymmetric CWCT is axially shifted off the middle plane, it can further enhance the damping of the $l=1$ modes. Damping effects on the $\mathrm{TM}_{011}$ mode, which usually has the highest shunt impedance and the lowest frequency among all HOMs, are shown in Fig. 5 as a function of the axial shift (W) off the middle plane. It is seen that with an axial shift of $30 \mathrm{~mm}$, the $\mathrm{Q}$ value drops down to $\sim 200$.
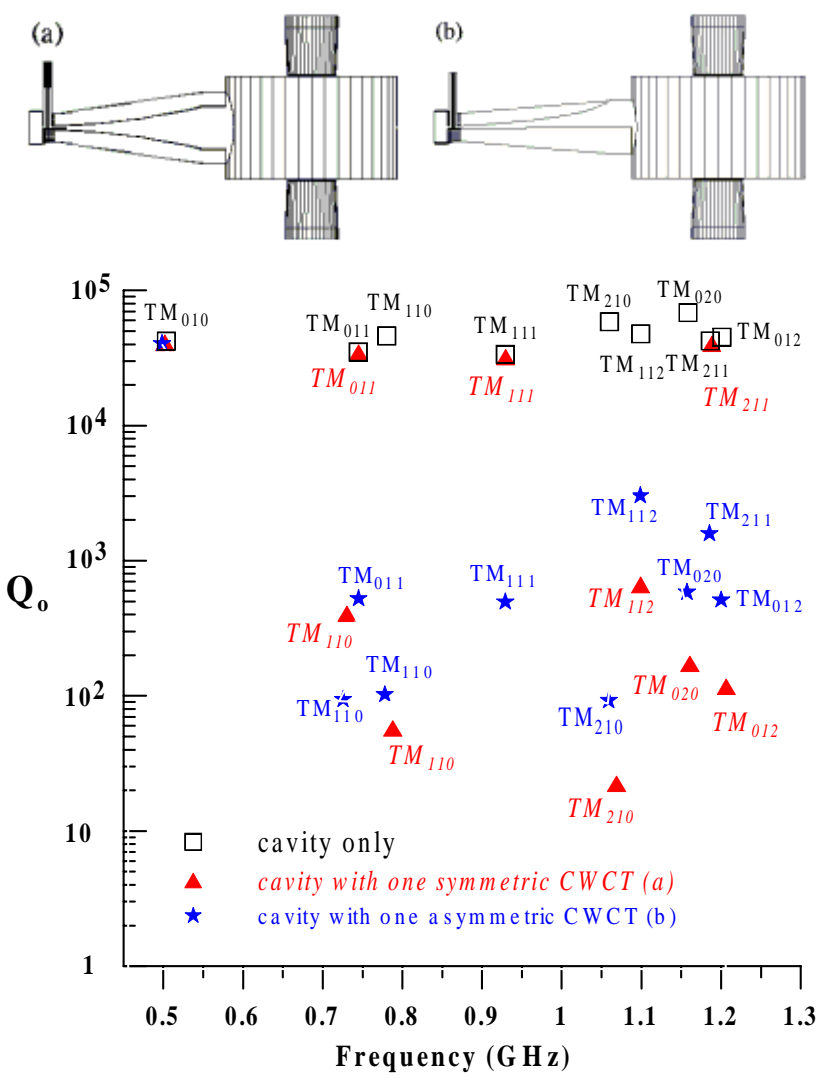

Fig. 4. Comparison of damping effects by the (a) symmetric and (b) asymmetric CWCTs, both placed in the middle of the sidewall of the DORIS cavity (shown on top figures).

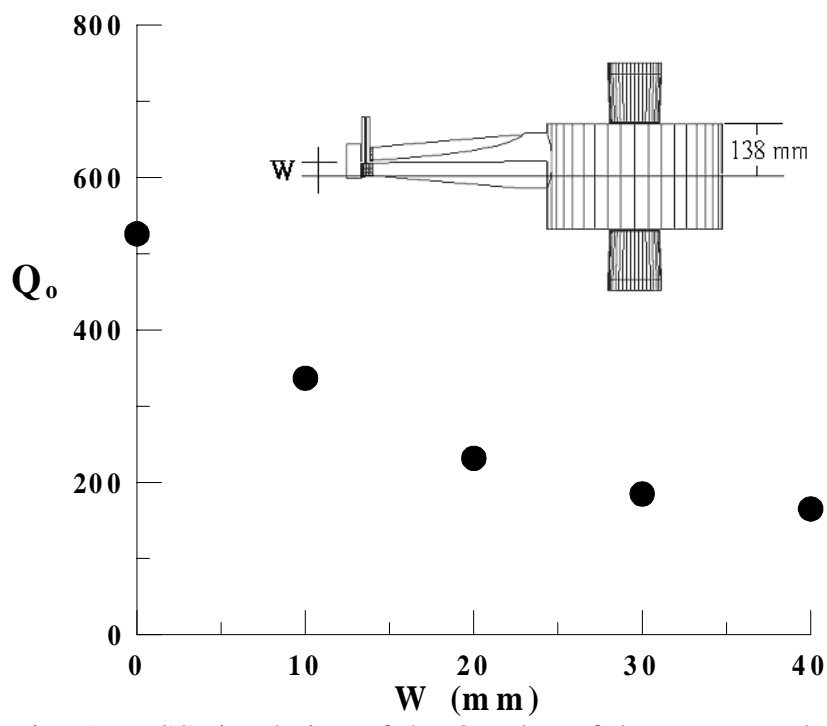

Fig. 5. HFSS simulation of the $Q$ value of the $\mathrm{TM}_{011}$ mode versus the axial shift (W) of the asymmetric CWCT.

Simulation results of the damping effects of an asymmetric CWCT shifted by $30 \mathrm{~mm}$ off the middle plane 
are shown in Fig. 6 and compared with those of an unshifted asymmetric CWCT. These results indicate:

1. The $l=0$ modes $\left(\mathrm{TM}_{010}, \mathrm{TM}_{110}, \mathrm{TM}_{210}\right.$, and $\left.\mathrm{TM}_{020}\right)$ are almost unaffected by the axial shift, as expected.

2. Damping of the $l=1$ modes $\left(\mathrm{TM}_{011}, \mathrm{TM}_{111}\right.$, and $\mathrm{TM}_{211}$ ) are significantly enhanced by the axial shift, again as expected.

3. Damping effects on both of the $l=2$ modes $\left(\mathrm{TM}_{112}\right.$ and $\mathrm{TM}_{012}$ ) are reduced by the axial shift. This is because the shift makes less field pattern matching between those HOMs of the cavity and the guiding mode of the asymmetric CWCT.

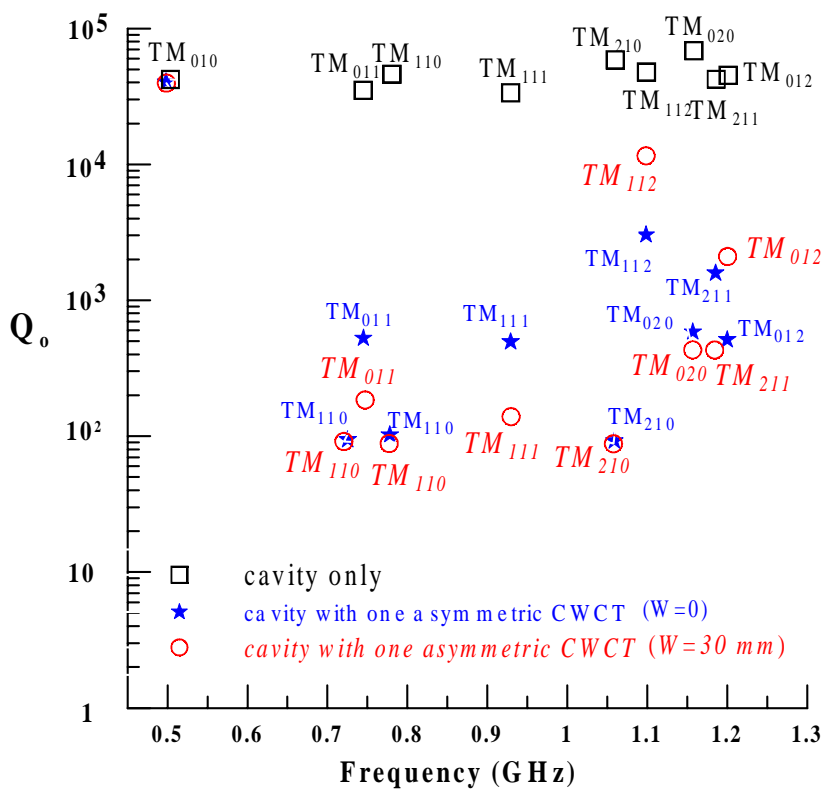

Fig. 6. Comparison of damping effects by an asymmetric CWCT located at $\mathrm{W}=0$ and $\mathrm{W}=30 \mathrm{~mm}$.

\section{CONCLUSION}

We have shown that the HFSS code can be a complimentary tool for calculating the resonant frequencies and the $\mathrm{Q}$ values of the cavity modes. The asymmetric CWCT is shown to be very effective in damping the $l=1$ modes in the DORIS cavity. The damping effects of $l=1$ modes can be further enhanced by slightly shifting the CWCT away from the middle plane of the cavity. However, damping effects on the $l=2$ modes (conceivably also the $l>2$ modes) by an asymmetric CWCT can be either enhanced or reduced. The overall merit of asymmetric CWCT versus that of the symmetric CWCT depends on the cavity configuration. A comprehensive study of the HOM damping of a high shunt impedance cavity by three symmetrically doubleridged waveguides including all $\mathrm{HOMs}$ up to $3 \mathrm{GHz}$ will be presented in a companion paper in this conference [8].

\section{REFERENCES}

[1] G. Conciauro and P. Arcioni, " A New HOM-Free Accelerator Resonator", Proc. $2^{\text {nd }}$ European Part. Acc. Conf., pp.149-151(1990).

[2] F. Schönfeld, E. Weihreter, R. Apel, and H. Henke, " A Cavity with Circular Waveguide for HOM Damping", Proc. $5^{\text {th }}$ European Part. Acc. Conf. , pp.1940-1942(1996).

[3] F. Schöfeld, E. Weihreter, Y.C. Tsai, and K.R. Chu, " Layout of a Broadband Circular Waveguide to Coaxial Transition", Proc. $5^{\text {th }}$ European Part. Acc. Conf., pp.1937-1939(1996).

[4] Y.C. Tsai, "Studies of High-Order-Mode Suppression in Storage Ring RF Cavities", Ph.D. Dissertation, National Tsing Hua University, 1997.

[5] E. Weihreter, S. Kuchler, Y.C. Tsai, and K.R. Chu, "Optimization and Experimental Characterization of a Broadband Circular Waveguide to Coaxial Transition", Proc. $6^{\text {th }}$ European Part. Acc. Conf., pp.20652067(1998).

[6] Y.C. Tsai, W.C. Wen, H.L. Cheng, C. Sung, Y.C. Huang, Ch.Wang, K.R. Chu and E. Weihreter, "Higher-Order-mode Damper Designs and Cavity Shape Optimization", HOM Damped Cavity Meeting, BESSY, Berlin, Germany, April 4, 2000.

[7] C.L. Hung, Y.C. Tsai, and K.R. Chu, " A Study of Open-End Cavities by the Field-Energy Method", IEEE Tran. Plasma Science, Vol. 26, No. 3, pp.931939(1998).

[8] F. Marhauser, E. Weihreter, D.M. Dykes, and P. McIntosh, “ HOM Damped 500 MHz Cavity Design for $3^{\text {rd }}$ Generation SR Sources", this conference. 